\title{
An oversampling system for ECG acquisition
}

\section{Yu Zhou}

School of Medical Instrument and Food Engineering, University of Shanghai for Science and Technology, Shanghai, China.

Email: Zyusst@yahoo.cn

Received 31 July 2009; revised 31 August 2009; accepted 3 September 2009.

\begin{abstract}
Traditional ECG acquisition system lacks for flexibility. To improve the flexibility of ECG acquisition system and the signal-to-noise ratio of ECG, a new ECG acquisition system was designed based on DAQ card and Labview and oversampling was implemented in Labview. And analog signal conditioning circuit was improved on. The result indicated that the system could detect ECG signal accurately with high signal-to-noise ratio and the signal processing methods could be adjusted easily. So the new system can satisfy many kinds of ECG acquisition. It is a flexible experiment platform for exploring new ECG acquisition methods.
\end{abstract}

Keywords: ECG Acquisition; Oversampling; DAQ; Labview

\section{INTRODUCTION}

ECG acquisition has been a mature technology since Doctor Willam Enthoven in Holland detected human ECG firstly. ECG can reflect the physiology status and pathology status of heart. Heart diseases can be detected in time by ECG. So ECG is used widely in clinic [1]. ECG can be divided into regular ECG, body surface potential mapping, high frequency ECG, ventricular late potentials electrogram, surface Hisbundle electrogram and etc. Their detection objects are all with low amplitude but distributing in different low frequency ranges [2]. Traditional ECG acquisition systems are designed according to different detection objects. Their analog signal conditioning circuit including multistage amplifiers and filters focus on special object. The disadvantage is that the special ECG acquisition system only can detect special object. So it lacks for flexibility and wastes resources.

The DAQ card and Labview produced by NI Company provide a flexible solution for data acquisition and processing $[3,4]$. DAQ card can detect signal accurately with high speed. And digital signal processing can be

Research supported by The Special Funds for Scientific Research for Selecting and Training Outstanding Young Teachers in Shanghai Universities (slg08029). implemented easily in Labview. There are many works using DAQ card and Labview in ECG. But they almost focus on the ECG's analysis and processing $[5,6]$.

To resolve the problem about flexibility, analog signal conditioning circuit is improved on and a new ECG acquisition system is developed with DAQ card and Labview.

Oversampling technology can exert the advantage of high speed A/D and improve the signal-to-noise ratio of signal detected. There has been detailed discussion about the use of oversampling in biopotential detection $[1,7,8]$. This technology is implemented in Labview and used in the new ECG acquisition system to improve the signalto-noise ratio of ECG.

\section{SYSTEM DESCRIPTION}

The oversampling system for ECG acquisition shown in Figure 1 is consisted of analog signal conditioning circuit, DAQ card and Labview.

Research supported by The Special Funds for Scientific Research for Selecting and Training Outstanding Young Teachers in Shanghai Universities (slg08029).

The analog signal conditioning circuit condition the ECG signal detected by electrodes to an analog signal with certain amplitude and frequency restriction. The analog signal is converted to digital signal by DAQ card with high speed sampling rate. And oversampling and other processing methods for the digital signal are implemented in Labview.

\section{ANALOG SIGNAL CONDITIONING CIRCUIT DESIGN}

Analog signal conditioning circuit included the circuit from the electrodes to A/D input of DAQ card. To fit for the need of the oversampling system for ECG acquisition, traditional circuit was improved on.

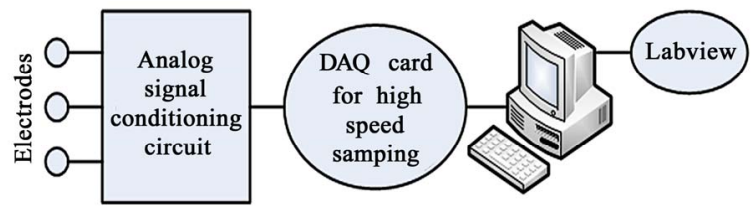

Figure 1. Lock diagram of the oversampling system for ECG acquisition. 


\subsection{Circuit Structure}

The circuit was shown in Figure 2. As traditional circuit, it included protection circuit, amplifying circuit based on AD620, high-pass circuit for eliminating polarization voltage, main amplifier, low-pass circuit and drivenright-leg circuit. But the parameters set of analog filter was different. In traditional circuit the filter parameters were set according to the object detected and couldn't be adjusted. Thus it lacked for flexibility. In oversampling system for ECG acquisition, the analog filter parameters were set with $0.03 \mathrm{~Hz}-4 \mathrm{KHz}$. Although the filter parameters were also fixed, the frequency range was wide and special digital filter parameters could be set further in Labview. This scheme guaranteed the currency of the system.

\subsection{Isolated Circuit and Power}

DAQ card and Labview worked based on PC. Isolated circuit was needed to protect the object detected from electric shock. HCNR200 was an optical coupler with high linearity and fit for the oversampling system for ECG acquisition. The isolated circuit based on HCNR 200 was shown in Figure 3. Isolated power supply was from 9V battery. LM2576 and ICL7660 got energy from $9 \mathrm{~V}$ battery and produced $\pm 5 \mathrm{~V}$. Non-isolated power supply was from DC supply.
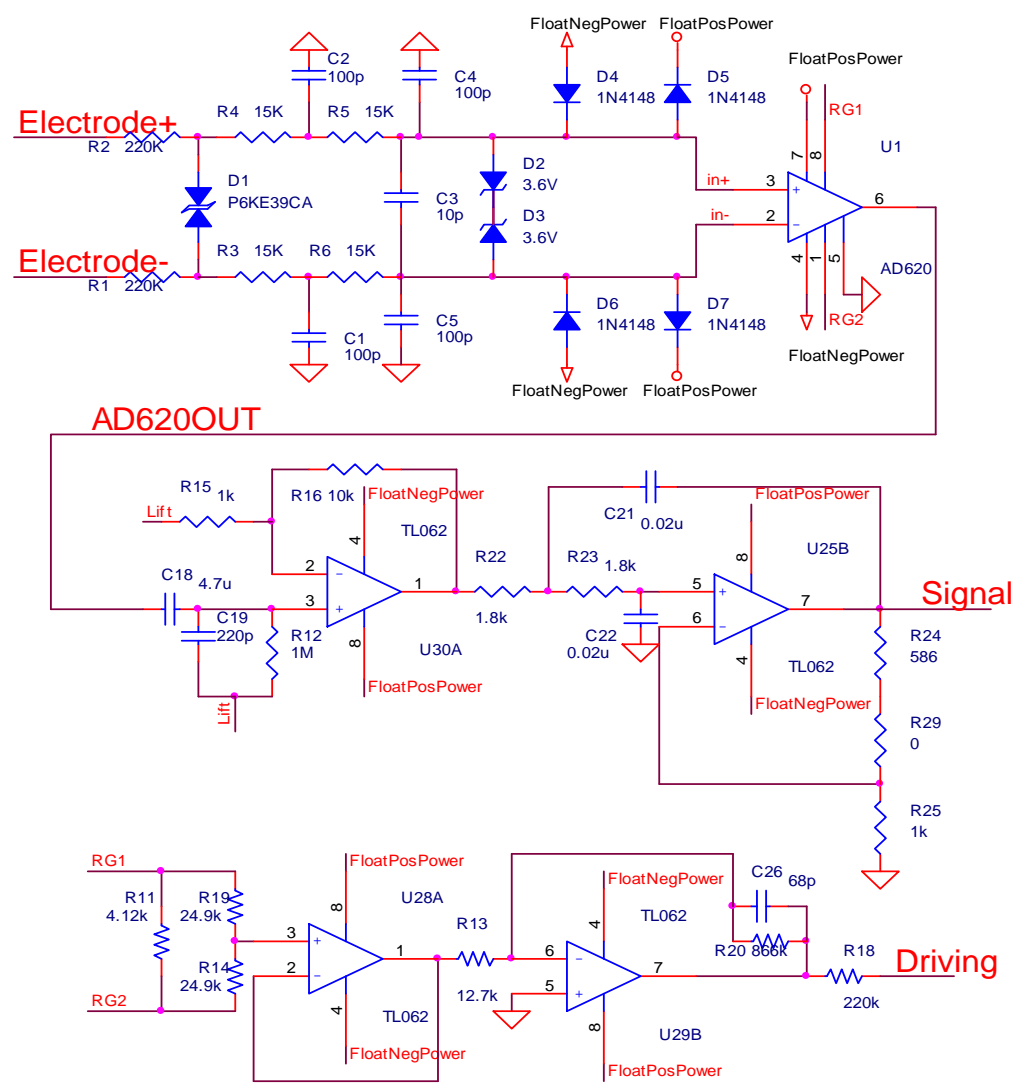

Figure 2. Analog signal conditioning circuit.

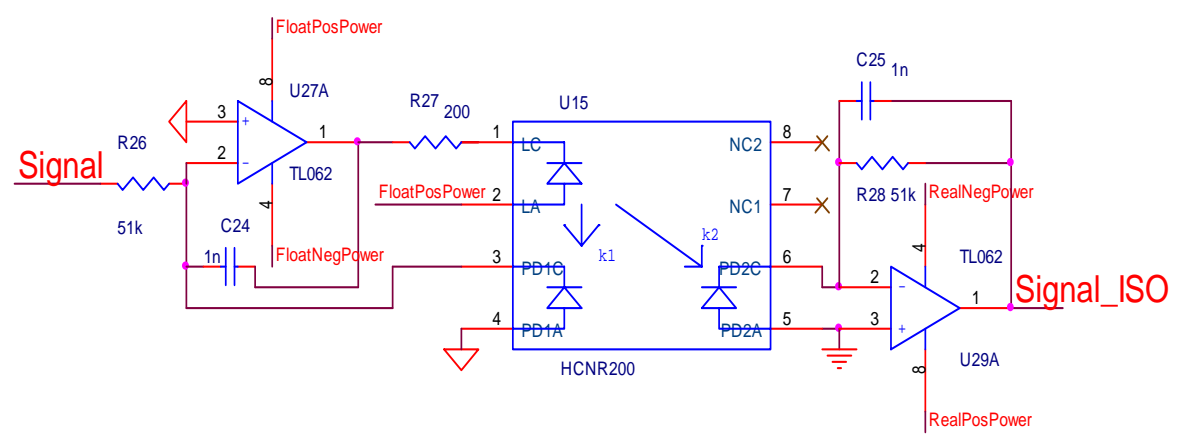

Figure 3. Isolated circuit. 


\section{LABVIEW PROGRAM DESIGN}

Labview program was the main body of the oversampling system for ECG acquisition. It included the set of DAQ card, digital filter, oversampling and etc.

\subsection{Setting DAQ Card}

DAQ6221 was a data acquisition card with PCI interface. Its highest sample rate for single channel was $250 \mathrm{KHz}$. In the oversampling system for ECG acquisition, the sample rate was set with $250 \mathrm{KHz}$ and continuous sampling mode was chosen. And the reference voltage for $\mathrm{A} / \mathrm{D}$ in DAQ card could be chosen. $\pm 5 \mathrm{~V}$ was set because the analog signal conditioning circuit had amplified the signal enough.

\subsection{Signal Filters}

Filters including low-pass filter and $50 \mathrm{~Hz}$ notch filter were needed in Labview. In the basis of analog filters with wide range, special digital filters were used for special object detected in Labview. It was the flexibility of the oversampling system for ECG acquisition. $50 \mathrm{~Hz}-$ interference caused by power line was an important problem in biopotential detection [9,10]. Notch filter was the tool to eliminate the interference. In Labview digital $50 \mathrm{~Hz}$ notch filter with more orders could get much better result than analog notch filter. At the same time the delay caused by digital filter was very small because of the high-speed cpu of PC.

In experiments, for low-pass filter, type was set with Butterworth, order was set with 50 and cut-off frequency was set with $90 \mathrm{~Hz}$. For notch filter, type was set with Bessel, order was set with 50 .

\subsection{Oversampling}

The criteria of using oversampling were that the original sample rate was high enough and the sample rate after oversampling could satisfy Nyquist sampling theorem.

The sample rate in DAQ card was set with $50 \mathrm{KHz}$. So the sample rate after oversampling was $50 \mathrm{KHz} / 256 \approx 195$ $\mathrm{Hz}$ if oversampling coefficient was set with 256. It was enough for ECG to satisfy Nyquist sampling theorem $[1,7]$. But if the oversampling coefficient was increased, the Nyquist sampling theorem couldn't be satisfied. If sampling rate was improved with 4 multiples, the resolution of ADC could be improved with 1 bit $[1,11]$. So the resolution of DAQ card' ADC was improved with 4 bits. Continuous sampling mode was chosen for DAQ card. So continuous 256 samples were sampled and accumulated and output. When this cycle went on, oversampling result was get. Oversampling coefficient and filter parameters were both decided by the object detected and could be set in Labview according to need. In experiments, $250 \mathrm{KHz}$ was also used for sample rate in DAQ card sometimes. Then the oversampling coefficient sh- ould be changed to 1024 and the sample rate after oversampling was $244 \mathrm{~Hz}$. It was also right for ECG.

\section{EXPERIMENTS DESIGNS AND RESULTS}

Some experiments were used to validate the design.

\subsection{Oversampling Experiment}

A sine wave with $20 \mathrm{~Hz}$ and $5 \mathrm{mV}$ Vpp similar to ECG produced by signal generator was input to DAQ card. The results were shown in Figure 4. Oversampling result was better than the direct sampling result apparently.

FFT was applied for the two waveforms in Figure 4 and the results were shown in Figure 5. The main com-

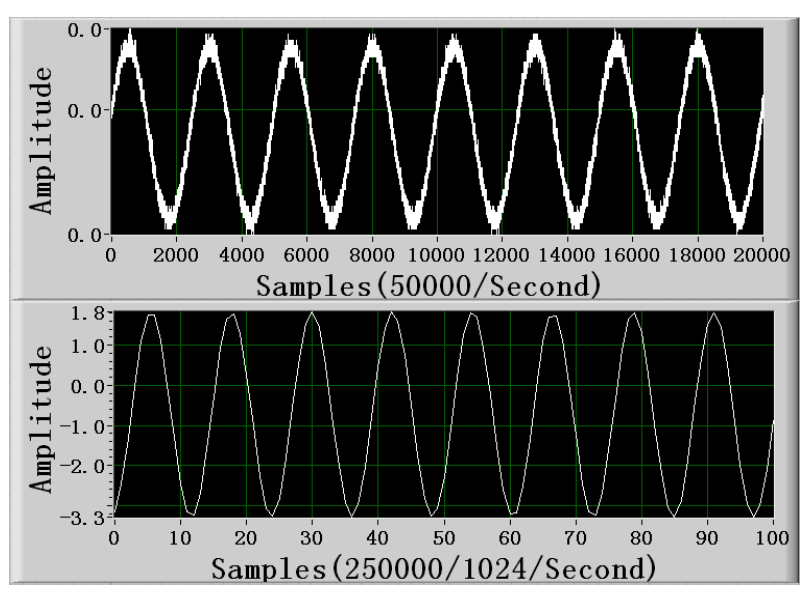

Figure 4. Direct sample result (upside, sampling rate: $50 \mathrm{KHz}$ ) vs. Oversampling result (downside, sample rate: $250 \mathrm{KHz}$, Oversampling coefficient: 1024).

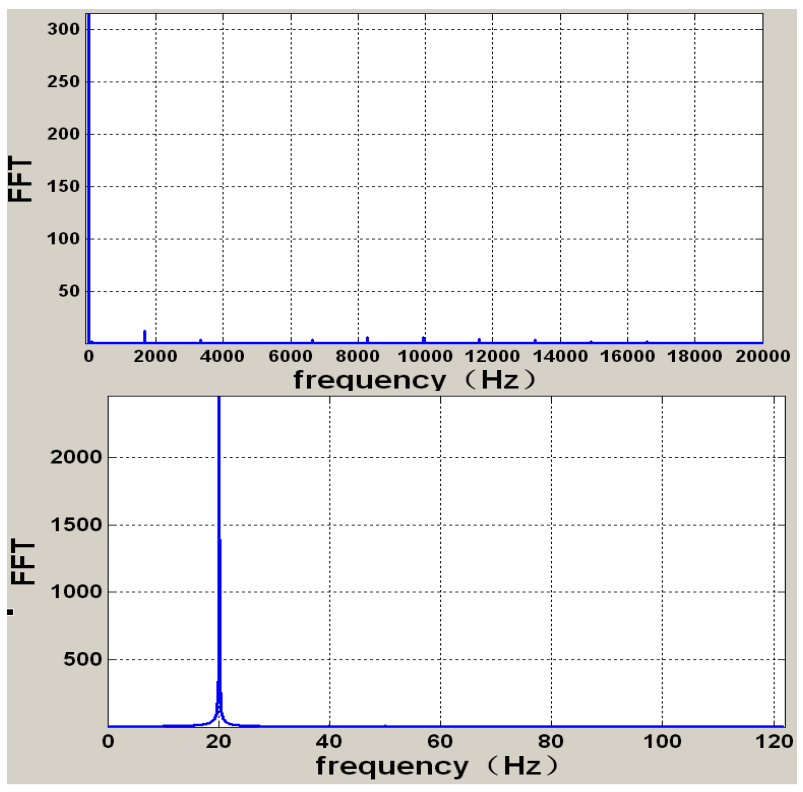

Figure 5. FFT for direct sample result (upside) vs. FFT for Oversampling result (downside). 
ponents in both FFT were in 20Hz. But there were many components in other frequencies in the FFT for direct sample result apparently. The FFT for oversampling result was clearer. Through careful observation, the value in $20 \mathrm{~Hz}$ in FFT for direct sample result was 316 and the value in $50 \mathrm{~Hz}$ which was the frequency of power line interference in FFT for direct sample result was 1.46. The corresponding values in FFT for oversampling result were 2454 and 10.3 respectively. Only considering these components, the signal to noise ratio were 216 for direct sample result and 238 for oversampling result. So the FFT for oversampling result was clearer and its signal to noise ratio was higher. These were the reasons that oversampling result was better than direct sample.

\subsection{ECG Acquisition Experiment with 3 Electrodes}

In the oversampling system for ECG acquisition, acquisition mode with 3 electrodes could be chosen where driven-right-leg electrode was included. Two electrodes for detection were pasted in the left wrist and right wrist respectively. The detection results were shown in Figure 6. The $1^{\text {st }}$ was direct sampling signal sampled by DAQ card, the $2^{\text {nd }}$ was signal after oversampling, the $3^{\text {rd }}$ was signal after low-pass filter and the $4^{\text {th }}$ was signal after low-pass filter and notch filter from upside to downside. It could be observed that the effect of the digital filters was good and oversampling could improve the signal-to-noise ratio of ECG. The components of ECG were shown clearly.

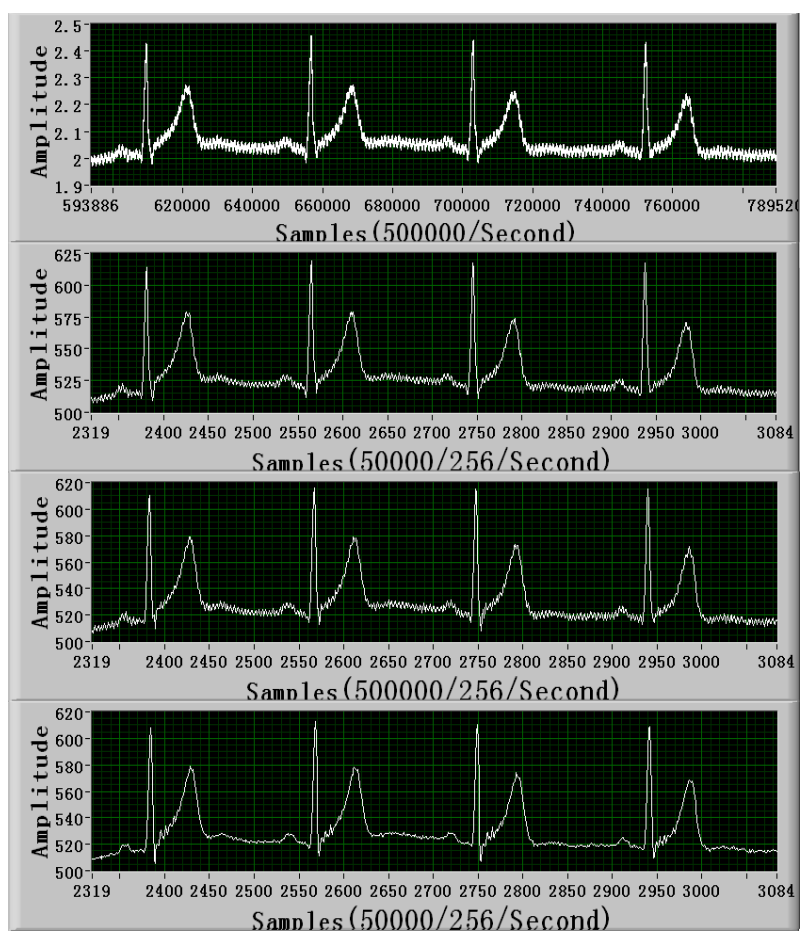

Figure 6. Sampling results with driven-right-leg circuit.

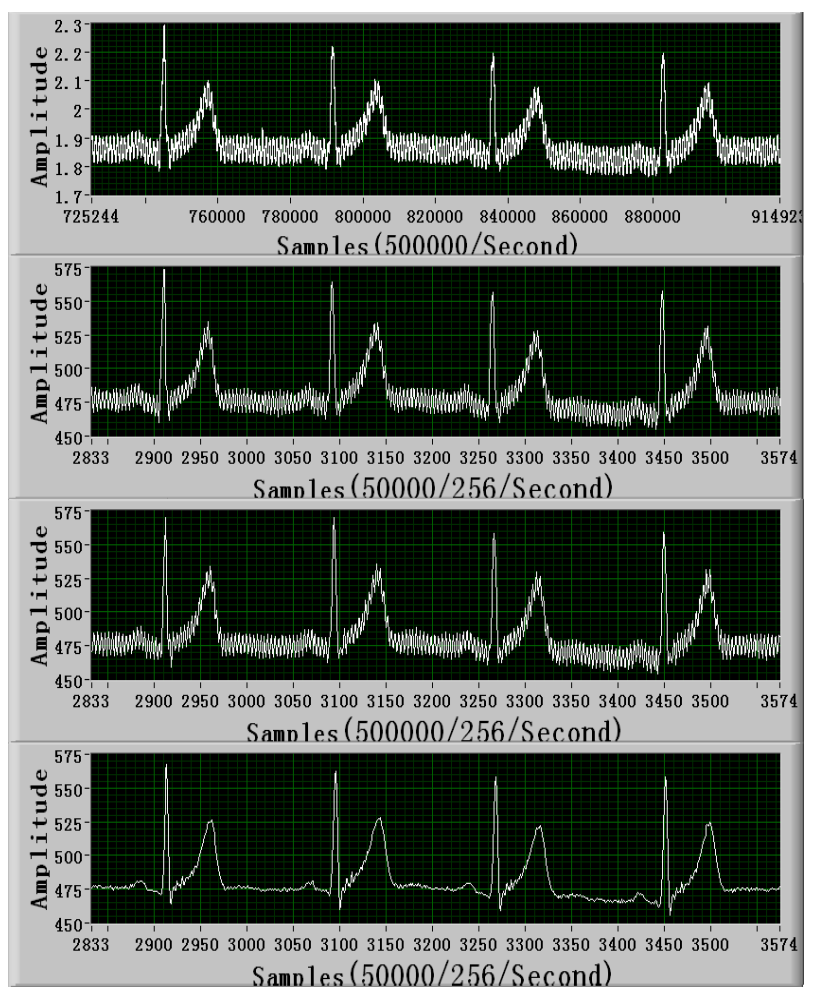

Figure 7. Sampling results without driven-right-leg circuit.

\subsection{ECG Acquisition Experiment with 2 Electrodes}

Also acquisition mode with 2 electrodes could be chosen for the oversampling system for ECG acquisition where driven-right-leg electrode was not included. Two electrodes were pasted in the left wrist and right wrist respectively. The detection results were shown in Figure 7. The $1^{\text {st }}$ was direct sampling signal sampled by DAQ card, the $2^{\text {nd }}$ was signal after oversampling, the $3^{\text {rd }}$ was signal after low-pass filter and the $4^{\text {th }}$ was signal after low-pass filter and notch filter from upside to downside. It could be observed that the power line interference was worse due to lack of driven-right-leg circuit before notch filter. But after notch filter ECG became very clear and the effect could be equivalent with the effect with drivenright-leg circuit. So profiting from digital filter with high orders, the detection result from 2 electrodes was satisfying. And oversampling could improve the signal-tonoise ratio of ECG further.

\subsection{ECG Acquisition Experiment in Left Arm}

In ECG monitoring field, it was a problem waiting to be resolved that how to acquire ECG with 2 electrodes expediently. The correlative experiment was attempted. An electrode was pasted in left wrist and the other was pasted in the left shoulder. The results were shown in Figure 8. It was observed that the ECG could be detected from one arm and oversampling made result a little clearer. 


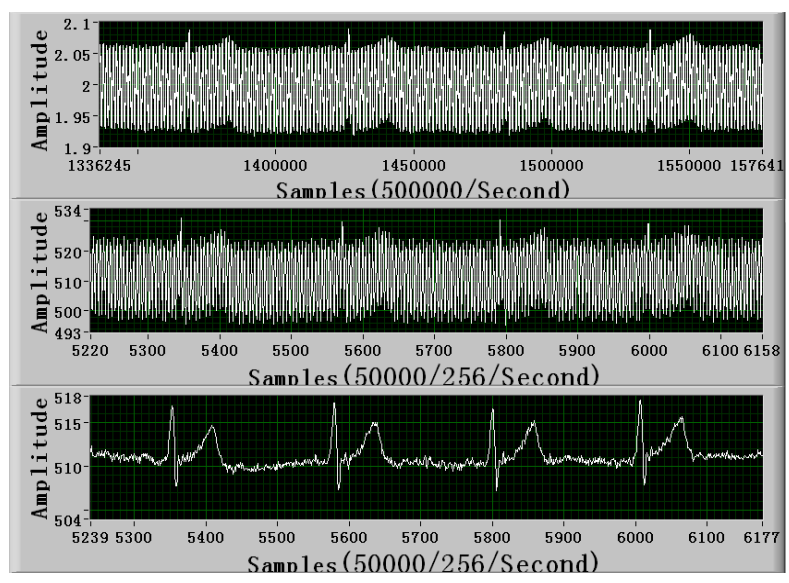

Figure 8. Sampling results from one arm (direct sample result (upside), Oversampling result (middle), result after oversampling and low-pass filter and notch filter (downside)).

\section{CONCLUSIONS}

The results of experiments indicate that the new oversampling system for ECG acquisition could detect ECG accurately, digital signal processing can be implemented easily in the system with DAQ card and Labview, ECG can be detected clearly with both 3 electrodes mode and 2 electrodes mode profiting from digital filters with high orders, oversampling improve the signal-to-noise ratio of ECG especially in one arm ECG detection. And the new system is flexible because the signal processing methods can be adjusted easily in Labview such as filter parameters and oversampling coefficient. So the oversampling system for ECG acquisition with flexible set and high signal-to-noise ratio can satisfy many kinds of ECG detection.

\section{REFERENCES}

[1] Li, G., Zhang, L. J., Lin, L., and He, F., (2008) Research of Bioelectricity Signal Detection Based on Oversampling, Acta Electronica Sinica, 36, 1465-1467.

[2] Wang, B. H., (2003) Biomedical Measurement and Instrument, 1st Edition, Fudan Press, Shanghai.

[3] Koutroulis, E. and Kalaitzakis, K., (2003) Development of an integrated data-acquisition system for renewable energy sources systems monitoring. Renewable Energy, 28, 139-152.

[4] Yeung, K. and Huang, J., (2003) Development of a remote-access laboratory: a dc motor control experiment. Computers in Industry, 52, 305-311.

[5] Wei, Y. C., Lee, Y. H., and Young, M. S., (2008) A Portable ECG Signal Monitor and Analyzer. The 2nd International Conference on Bioinformatics and Biomedical Engineer- ing. Shanghai, China, pp.1336-1338.

[6] Yeon, N., Sung, P., Kyu, H., et al., (2006) A Study of Significant data Classification between EDR extracted and frequency analysis of Heart Rate Variability from ECG using Conductive textile. World Congress on Medical Physics and Biomedical Engineering. Seoul, Korea, pp.4100-4103.

[7] Li, G., Zhang, L. J., Lin, L., and He, F. (2008) Weak Signal Detection Based on Over-Sampling and Saw-Tooth Shaped Function. ACTA ELECTRONICA SINICA, 36, 756-759.

[8] Goes, J., Paulino, N., Pinto, H., Monteiro, R., Vaz, B., and Garcao, A. S., (2005) Low-power low-voltage CMOS A/D sigma-delta modulator for bio-potential signals driven by a single-phase scheme. Circuits and Systems I: Regular Papers, IEEE Transactions on, 52, 2595- 2604.

[9] Huhta, J. C. and Webster, J. G. (1973) 60-Hz Interference in Electrocardiography. IEEE Transactions on Biomedical Engineering, 20, 91-101.

[10] Spinelli, E. M. and Mayosky, M. A., (2005) Two-electrode biopotential measurements: power line interference analysis. Biomedical Engineering, IEEE Transactions on, 52, 1436-1442.

[11] Oppenheim, A. V., Schafer, R. W., and Buck, J. R., (1999) Discrete-time Signal Processing, 2nd Edition, Prentice Hall, New Jersey. 